\title{
GUEST EDITORIAL Editors may inappropriately influence authors' decisions regarding selection of references in scientific articles
}

International Journal of Impotence Research (2007) 19, 443-445; doi:10.1038/sj.ijir.3901583

The journal impact factor has been invented as an index of evaluation of the impact and thus, by inference, the quality of scientific journals. However, even the inventors of this index admit that it has found uses beyond their initial intention. Given the various implications of the impact factor including financial ones, most journal publishers and editors have become keen to increase the impact factor of their journal(s). Thus, they use various strategies in achieving this goal. We have noted that, fortunately, a minority of journals have used a particular strategy of boosting the impact factor with which we completely disagree. Specifically, editorial instructions to authors, usually in the editor's letter requesting revision of a manuscript under consideration for publication, may include suggestions that authors cite in their manuscripts articles published in the editor's journal, without specifying relevant publications that may be indeed overlooked by the authors. We believe that this strategy of increasing the impact factor is not correct because it interferes with authors' decisions regarding the selection of the most appropriate citations to previous work for inclusion in their manuscript. This practice should be abandoned by the journals that use it before it becomes an epidemic that will affect the majority of scientific journals.

The right to receive credit for intellectual work and new ideas is a major principle in the academic community. However, there is no debate regarding the fact that even the best and breakthrough original research is inevitably founded, to some extent, upon earlier scientific work. ${ }^{1}$ Thus, the practice of citing of previous work in the reference list of a scientific article is essential in attributing the appropriate credit to authors of relevant articles. Most publishers, editors, authors and readers consider any departure from this practice as scientifically unethical. It was a historic phrase by Newton, 'if I have seen further, it is by standing on the shoulders of giants', that set the ethics and moral context for the use of citations in all scientific works.

However, beyond the scientifically ethical point of view, references have been attributed with several other more practical roles. The usually lengthy section of references generally found in academic papers permits the reader to further refer to more extensive bibliography, erasing at the same time unnecessary details and keeping the main text of an article as short as possible and sharp to the point.

References may be used to support or refute the statements included in the main body of an article. Citing an authoritative piece of writing recognized as landmark contribution for the field is perhaps the best way to make a strong point. ${ }^{2}$ Furthermore, references permit the citation of opinions and ideas of scientists with major contribution in the field without disturbing the flow of the manuscript and the integrity of the original ideas proposed by the authors. ${ }^{2}$ Consistent or not with the findings of previous research is challenging and helps science evolve and discover answers to the questions.

With sadness, we see that the reference section of a scientific paper has become a point with various interests, unfortunately in a different sense than its original royalty-delivering purpose and ethical value. Specifically, citations to previous work are used for the calculation of the impact factor of scientific journals. Both scientists and journal editors have such considerable respect to the index of quality of scientific journals. The impact factor, developed by the Institute of Scientific Information (ISI)—now a part of Thomson Scientific, is an index that initially aimed, when conceived, to serve as a measure of quality for scientific publishing that could help in decisions regarding inclusion of a journal in the indexing databases. However, it has accumulated such power that is used today inappropriately, even based on the opinion of its creator, ${ }^{3}$ to judge beyond journals' quality, scientists' efficiency for academic positioning, grant awarding and governmental funding policies. ${ }^{4}$

Thus, it comes without surprise that journals seek to increase their impact factor figure to enhance their influence in the scientific community. In addition, commercial publishers strive to win a larger part of the publishing market and subsequently some of them may request from editors to try to increase the impact factor of their journal. Also, the executive boards of regional, national or international professional societies may also consider that a high impact factor journal adds more credibility and prestige to their society.

The great majority of editors are aware of the impact factor game' and how it is best played., However, only a minority of them seems to be willing to engage in such an activity ${ }^{5}$ that mainly 
serves business and marketing purposes rather than pure advance of science.

The journal impact factor during a specific year is defined by the following equation: the number of citations in the specific year, to articles published in the specific journal during the 2 precedent years, divided by the number of citable articles published in the specific journal during the 2 precedent years. ${ }^{7}$ The determination whether a publication is included in the group of 'citable articles' and, thus, in the denominator of the impact factor calculation is made exclusively by ISI. Several types of publications, including letters to the editor and commentaries and editorials without an abstract, are not usually included in the group of 'citable articles'. The relevant calculations for the derivation of the impact factor of about 8700 journals in the various scientific fields (Science, Technology, Social Sciences, and Arts and Humanities) that are tracked by ISI are made by this company. The new impact factors of the various journals are usually announced in June of each year and are expected with great interest by most publishers and editors as well as a considerable minority of authors of scientific papers.

The calculations involved in the determination of the impact factor of each journal include, as it explained above, the citations to articles published in the specific journal. Taking this into consideration, anyone may understand why the reference section of scientific publications is associated with various attributes, including a considerable financial meaning.

The journal impact factor calculations received heavy critique for being arbitrary and insufficient and many authorities dispute the ranking of journals. ${ }^{8,9}$ We do not intend to further judge this tool as an index of quality of scientific journals because this is out of the scope of this article. Instead, below we will try to focus and discuss on one of the most questionable practices of engineering and manipulating the journal impact factor that is undertaken by some journals.

Publishers and editors of journals may employ several strategies in their effort to increase their impact factor. ${ }^{5,6}$ In brief, we think that the editorial policies that may influence the impact factor of a specific journal can be divided into two major categories. The first includes practices that may not be necessarily criticized; these may include the setting of high-quality standards for the acceptance of publications, the selection of publications originating from quality scientists internationally recognized for their work, the focus on hot areas of high research activity and interest, and the reluctance to publish studies with negative or confirmatory results. Many authors and editors question the scientific value of such policies, especially the reluctance by some journals to publish studies with negative results that lead to under-representation of such studies, and thus to a biased overall scientific literature. However, we think that someone should not criticize the efforts of editors to increase the quality of their journals by using various methods including the selection of the most promising articles for peer review, the selection of referees that are able and willing to evaluate the article and suggest areas of improvement, and finally the suggestion of editorial points that enhance the clarity and accuracy of statements made in a manuscript.

In the second category of editorial policies that may influence the impact factor of a specific journal, someone may include policies that have the potential to distort the true image of the journal's contribution by enchasing the impact factor and thus altering the relevant scientific field journal rankings. Increasing the number of publications of the types of articles that are not included in the denominator of the impact factor equation like a vivid correspondence section, boosting self-citation through editorials and special articles and sometimes whole journal issues that summarize past publications of the journal, favoring submission of review articles and technical reports that are citation attracting and denying other types of invaluable publications such as educational case reports and pictorial essays that are unlikely to be cited, are only few of the 'tools' that publishers and editors use to increase the impact factor of their journal. ${ }^{10}$

However, perhaps the most noteworthy editorial policy regarding the impact factor manipulation is to suggest or even require that authors cite in their manuscripts articles published in the editor's journal or other journals belonging to the affiliate publishing consortium. Instructions of this type are included in the editor's letter, usually when requesting revision of a manuscript for re-consideration for publication in the journal. ${ }^{11,12}$ Fortunately, only a minority of journals employs this practice. By using this technique, journals aim to achieve a higher rate of self-citation and, thus, artificially boost their impact factor and order in the relevant scientific field journal ranking. We know that this is a practice that has bothered, some would say offended, several authors from time to time and is a common secret in the scientific community. However, few researchers are willing to go public with such allegations. ${ }^{11}$ We would like to openly express our disagreement with this practice and suggest to the minority of journal editors and publishers that use this practice to abandon it.

Given the importance of the references section in a scientific article, the selection and use of citations to previous publications should be based on specific criteria and, thus, made with scrutiny. Original research publications with efficient and reproducible methodology as well as recognized scientific contribution that represent up-to-date knowledge and are focused to the field of interest should be 
selected to support the statements of the author(s). We believe that trying to inappropriately influence authors' decisions regarding selection of references may cancel their meaning and significance. The author is solely responsible for the selection of the references that will properly support his/her arguments and will add to the soundness and credibility of the work. ${ }^{1}$ Only an independent peer reviewer who believes that a relevant landmark contribution for the field has been overlooked by the author(s) may address instructions for inclusion of specific references. Such revision of a manuscript is fully justified, supportive for the author and invaluable for the reader.

We emphasize again that our view is that editors are not entitled to ask authors for revision of the references section to the benefit of their own journal's impact factor. We believe that most scientists submitting their work for consideration for publication would not really like to make chances in the reference section in favor of the journal's impact factor with the suggestion of the editor. However, they may feel awkward when they receive such suggestions with the editor's request for revision of the manuscript. In fact, most authors would comply with such a request for various reasons, even if they feel that this practice is questionable. We believe that it is mainly the responsibility of the publishers and the editors not to use such practices that may influence authors' choices in selecting the appropriate citations to previous work for inclusion in their manuscript.

Although it is sometimes said that such incidences are just 'ludicrous extremes' of the journal impact factor manipulation strategies, ${ }^{12}$ we believe that this particular practice should be simply and fully abandoned by all journals that use it. From our experience, we can tell that they are certainly not rare exceptions even among the group of wellrespected, prestigious biomedical journals. Such efforts should be brought to public scrutiny and be condemned as a whole by the scientific community. It should be mentioned that authors also might use inappropriately a large numbers of citations to their own previous work. ${ }^{13}$ This is another important problem that sometimes interferes with the selection of the most appropriate references in a scientific article. Although this practice should be also criticized and avoided, the serious issue of author self-citations is out of the scope of this article.

Many scientists talk about the need of an unbiased index that measures publications' quality and

impact. No matter the index used, including the ISI impact factor or various derivations from calculations of data from other databases such as Google Scholar or CrossRef, there will always be ways to manipulate the various adopted indices of journal quality and engineer false impressions. We believe that publishers and editors of scientific journals have the right to use one or more of the available indices of evaluation of journal quality and impact for various reasons, including marketing purposes. However, we think that the practice of providing editorial suggestions that authors cite in their manuscripts articles published in the editor's journal is not correct. This practice should stop before it becomes an epidemic that will affect the majority of scientific journals.

Conflict of interest: None for both authors.

Funding: None.

ME Falagas ${ }^{1,2}$ and VG Alexiou ${ }^{1}$

${ }^{1}$ Alfa Institute of Biomedical Sciences (AIBS),

Athens, Greece and ${ }^{2}$ Department of Medicine, Tufts

University School of Medicine, Boston, MA, USA

E-mail: m.falagas@aibs.gr

\section{References}

$1 \mathrm{McD}$ Taylor D. The appropriate use of references in a scientific research paper. Emerg Med (Fremantle) 2002; 14: 166-170.

2 Eunson B. Writing and Presenting Reports, 1st edn. John Wiley and Sons: Singapore, 1994.

3 Garfield E. Journal impact factor: a brief review. CMAJ 1999; 161: 979-980.

4 Moed HF, Van Leeuwen TN. Impact factors can mislead. Nature 1996; 381: 186.

5 Editorial. The impact factor game. It is time to find a better way to assess the scientific literature. PLoS Med 2006; 3: E291.

6 Rogers LF. Impact factor: the numbers game. AJR Am J Roentgenol 2002; 178: 541-542.

7 Garfield E, Sher IH. Genetics Citation Index. Institute for Scientific Information: Philadelphia, PA, 1963.

8 Hecht F, Hecht BK, Sandberg AA. The journal 'impact factor': a misnamed, misleading, misused measure. Cancer Genet Cytogenet 1998; 104: 77-81.

9 Opthof T. Sense and nonsense about the impact factor. Cardiovasc Res 1997; 33: 1-7.

10 Seglen PO. Why the impact factor of journals should not be used for evaluating research. BMJ 1997; 314: 498-502.

11 Hemmingsson A, Mygind T, Skjennald A, Edgren J. Manipulation of impact factors by editors of scientific journals. (letter) AJR 2002; 178: 767.

12 Rogers LF. Manipulation of impact factors by editors of scientific journals. (reply to letter) AJR 2002; 178: 767.

13 Falagas ME, Kavvadia P. 'Eigenlob': self-citation in biomedical journals. FASEB J 2006; 20: 1039-1042. 